\title{
An Infrastructure for Workplace Learning Analytics: Tracing Knowledge Creation with the Social Semantic Server
}

\author{
Adolfo Ruiz-Calleja ${ }^{1 *}$, Sebastian Dennerlein ${ }^{2}$, Dominik Kowald², Dieter Theiler ${ }^{2}$, Elisabeth \\ Lex ${ }^{2,3}$, Tobias Ley ${ }^{4}$
}

\begin{abstract}
In this paper, we propose the Social Semantic Server (SSS) as a service-based infrastructure for workplace and professional learning analytics (LA). The design and development of the SSS have evolved over eight years, starting with an analysis of workplace learning inspired by knowledge creation theories and their application in different contexts. The SSS collects data from workplace learning tools, integrates it into a common data model based on a semantically enriched artifact-actor network, and offers it back for LA applications to exploit the data. Further, the SSS design's flexibility enables it to be adapted to different workplace learning situations. This paper contributes by systematically deriving requirements for the SSS according to knowledge creation theories, and by offering support across a number of different learning tools and LA applications integrated into the SSS. We also show evidence for the usefulness of the SSS extracted from 4 authentic workplace learning situations involving 57 participants. The evaluation results indicate that the SSS satisfactorily supports decision making in diverse workplace learning situations and allow us to reflect on the importance of knowledge creation theories for this analysis.
\end{abstract}

\section{Notes for Practice}

- We propose the Social Semantic Server (SSS) as a service-based infrastructure for workplace and professional learning analytics (LA) that focuses on knowledge creation theories.

- We identify the requirements for the SSS and present its design and development.

- We evaluated the SSS by integrating a set of learning tools and LA applications into the SSS and using it in 4 authentic workplace learning situations involving 57 participants.

\section{Keywords}

Learning analytics, informal learning, workplace learning, artifact-actor network, data infrastructure

Submitted: 28.06.2018 - Accepted: 01.02.2019 — Published: 05.08.2019

Corresponding author ${ }^{1}$ Email: adolfo@tlu.ee Address: School of Digital Technologies, Tallinn University, Narva mnt 25, 10120, Tallinn, Estonia

${ }^{2}$ Address: Know-Center GmbH. Research Center for Data-Driven Business and Big Data Analytics, Inffeldgasse 13, 6th floor, $8010 \mathrm{Graz}$, Austria

${ }^{3}$ Address: Graz University of Technology. Institute of Interactive Systems and Data Science, Inffeldgasse 13, 5th floor, 8010 Graz, Austria

${ }^{4}$ Address: School of Educational Sciences, Tallinn University, Narva mnt 25, 10120, Tallinn, Estonia

\section{Introduction}

Workplace and professional learning happens across a multitude of formal and informal settings where professionals advance their competence, mostly in a self-directed manner. Workplace learning can be a rather informal way of gaining knowledge and expertise by self-directed exploration and social exchange that is tightly connected to the processes and the places of work (Eraut, 2004). In contrast to formal education, workplace learning is often driven by personal interest or by problems that appear in the work context. It typically lacks a pedagogical design to guide the learning process (Kooken, Ley, \& De Hoog, 2007). While professionals are also involved in more formal learning settings, such as training, they are commonly motivated by job-based demands and the need to contribute to workplace performance. The fact that workplace learning is multi-episodic, happens across diverse contexts, and is tightly coupled with the workplace poses several challenges for the design and development of technology that supports and analyzes workplace learning (Klamma, 2013).

In this paper, we address challenges related to learning analytics (LA) in workplace settings. LA collects data about learning processes and feeds it back to learners or trainers to support their decisions about their own or others' learning. LA in the 
workplace faces a number of challenges (Cardinali, 2015; Ruiz-Calleja, Dennerlein, Ley, \& Lex, 2016; Ruiz-Calleja, Prieto, Ley, Rodríguez-Triana, \& Dennerlein, 2017). For example, learners use a number of learning tools in a spontaneous and difficult-to-foresee way because learning does not follow a planned curriculum or pedagogical design. A number of such learning tools have been proposed to support specific workplace learning tasks, such as the creation of portfolios that allow learning to be traced in multiple contexts (Krull \& Leijen, 2015), or peer discussions to support help-seeking (Santos et al., 2016). However, if we want to look at workplace and professional learning processes across different tools, contexts, and learning tasks, then LA needs to take a more holistic perspective.

To provide this more holistic picture for workplace LA, we need to coherently analyze data from several tools used for learning in the workplace. Some LA infrastructures have been proposed to collect, integrate, and process data from several learning tools. While some of these proposals have been designed and tested in realistic situations (e.g., Renzel \& Klamma, 2013; Siadaty et al., 2012)), most of them still focus on a limited number of learning tasks and tools. Additionally, a holistic perspective requires us to rely on a careful analysis of existing learning theories, one of the major challenges in the LA community (Gašević, Dawson, \& Siemens, 2015). For workplace and professional learning, this is even more critical because there is no curriculum or pedagogical design to guide the analysis. Thus, a focus on a particular learning theory is crucial to guide the processes of collecting, managing, and representing workplace learning data. Much too often, theoretical claims remain implicit.

In a recent review, we analyzed existing proposals for workplace LA (Ruiz-Calleja et al. 2017). This analysis leads us to conclude that most current proposals focus on theories that follow knowledge acquisition or participation metaphors (Paavola \& Hakkarainen, 2005). In these cases, individuals are understood as the basic unit of knowing and learning (knowledge acquisition), or learning is seen as an interactive process of participating in cultural practices (participation). We see much less focus on the knowledge creation metaphor (Paavola \& Hakkarainen, 2005), which considers learning as a joint development of objects of activity. This is especially true for LA infrastructures that allow us to trace learning processes across several learning tools and contexts. This constitutes a significant problem because it means missing essential elements of a learning situation, such as how new knowledge is created or how innovation processes happen in communities. Considering that learning should focus on innovation, creative problem solving, and knowledge creation (Peschl \& Fundneider, 2014) in order to keep a competitive edge in the current knowledge-based economy, this missing emphasis on the knowledge creation metaphor in LA is especially troublesome.

To address these limitations, we propose to exploit the Social Semantic Server (SSS) (Dennerlein, Kowald, et al., 2015) as an infrastructure for workplace LA. This paper systematically derives the SSS requirements according to knowledge creation theories, with a special focus on how data from different learning tools is coherently combined and offered back to LA applications. We also illustrate the support offered by the SSS across a number of different learning tools and settings and collect evidence for its usefulness from four evaluation studies. These studies allowed us to reflect on the importance of knowledge creation theories for workplace LA.

The rest of the paper is structured as follows: first we summarize the state of the art related to workplace LA infrastructures; then we describe the SSS, whose evaluation is then presented and discussed; we end the paper by summarizing its conclusions.

\section{Data Infrastructure for Workplace LA}

The field of workplace LA is still in its early stages of development, but interest in it has increased in the last few years (Ruiz-Calleja, Prieto, Ley, Rodríguez-Triana, \& Dennerlein, 2017). Some LA projects, such as LACE ${ }^{1}$, moved their attention to the workplace domain (Cardinali, 2015), and other workplace learning projects, such as Learning Layers ${ }^{2}$, began to use LA to analyze and support learning processes (Ruiz-Calleja, Dennerlein, Ley, \& Lex, 2016). These projects exploited LA techniques as a way to assess or support decision making in workplace learning processes. These proposals are implicitly or explicitly grounded by particular learning theories (Gašević, Dawson, \& Siemens, 2015). We reviewed them following the three metaphors of learning — knowledge acquisition, participation, and knowledge creation — defined by Paavola \& Hakkarainen (2005). These metaphors can be understood as different lenses for the design or analysis of learning situations and are "closely connected to the way knowledge is understood in different conceptions of learning" (Paavola \& Hakkarainen, 2005). The metaphors help us to understand the assumptions that guide the creation of existing LA applications and infrastructures, especially those assumptions related to how knowledge is represented.

Many LA proposals follow the knowledge acquisition metaphor. This metaphor assumes individuals as the basic unit of learning. Hence, these LA applications commonly model the learners according to the knowledge they acquired (e.g., Ley \& Kump, 2013; Niemann \& Wolpers, 2014). Depending on the learning tools, this knowledge may be stated as a set of competencies (e.g., Krull \& Leijen, 2015) or as a set of topics for which the learner is considered an expert (e.g., Ley \& Kump,

\footnotetext{
${ }^{1}$ http://www. laceproject.eu/

${ }^{2}$ http://learning-layers.eu
} 
2013). These LA systems typically use ontologies to structure the data they manage (e.g., Nussbaumer et al., 2012; Siadaty, Gašević, \& Hatala, 2016b) or other formal conceptualizations of the learning domain, such as knowledge spaces (e.g., Ley \& Kump, 2013).

Other proposals (e.g., Rajagopal, van Bruggen, \& Sloep, 2017; Buckingham-Shum \& Ferguson, 2012) follow the participation metaphor, which assumes that learning happens by participating in cultural practices that shape cognitive activity in manifold ways. These LA applications focus on modelling learning communities and groups depending on their social behaviour. Therefore, they create social networks to abstract the social interactions that occur in the tool. In many cases, social network analysis techniques are employed to extract the community's expertise about certain topics or to detect communities (Klamma, 2013) and unconnected subnetworks in professional networks (e.g., de Laat \& Schreurs, 2013).

Other examples (e.g., Derntl, Günnemann, \& Klamma, 2013; Southavilay, Yacef, Reimann, \& Calvo, 2013) can be found that follow the knowledge creation metaphor, which deals with the collaborative and systematic development of common objects of activity. These LA applications model how learning materials and conceptual artifacts are collaboratively created (Schoefegger, Seitlinger, \& Ley, 2010; Thüs, Chatti, Brandt, \& Schroeder, 2015). The group of learners is taken as the unit of analysis, considering also their tools and common artifacts (Berendt, Vuorikari, Littlejohn, \& Margaryan, 2014; BuckinghamShum \& Ferguson, 2012). Hence, interactions between learners and artifacts and the contexts in which these interactions happen are taken into account, creating a context-aware artifact-actor network (AAN) (Ruiz-Calleja, Dennerlein, Tomberg, Ley, et al., 2015), which is then exploited to understand the evolution of the learners and artifacts (e.g., Fidalgo-Blanco, Sein-Echaluce, Garcia-Penalvo, \& Conde, 2015; Thüs, Chatti, Brandt, \& Schroeder, 2015). It is typical for such systems to make use of folksonomies, enabling users to introduce new and unexpected terms or topics (Schmidt et al., 2009). The number of LA proposals that follow this metaphor is much lower than in the previous two metaphors. This is surprising because of the long history of knowledge creation theory (Nonaka, 1994) and its recognized importance in workplace learning and professional development in the knowledge society (Paavola \& Hakkarainen, 2005).

Another restriction shared by all of the LA proposals presented above is that they only collect and process data from a single application. Consequently, these proposals put less emphasis on the reusability of the data they manage and the algorithms employed to manipulate this data. However, the LA vision goes beyond these restrictions (Siemens et al., 2011): learners, especially workplace learners, typically employ several tools in a way that is difficult to foresee. Researchers are therefore encouraged to develop open proposals that enable the integration of content and data from different sources. These proposals should also be extensible for third parties to integrate their own data sources and data processing techniques. Following this vision, several LA infrastructures were proposed to enhance the integration of data from several tools as well as the reusability of this data and the algorithms to process it (Duval, 2011) (see Table 1). Next, we will review these proposals.

Several authors propose infrastructures that exploit the data collected by learning management systems (LMSs) in formal learning contexts. LMSs successfully integrate data from different tools, but they structure learning processes according to a pre-described pedagogical design that does not commonly exist in workplace learning. Nonetheless, some authors exploit the data collected by LMSs for analysis that goes beyond the pedagogical design. For example, Fidalgo-Blanco et al. (2015) use Moodle data to assess the individual contributions in teamwork activities. For this purpose, they analyze the relationships between learners and between learners and learning artifacts inside each team. They focus on a set of indicators to understand and assess how the team collaborated. Another very interesting proposal is the Connected Learning Analytics (CLA) toolkit (Bakharia, Kitto, Pardo, Gašević, \& Dawson, 2016). A distinguishing characteristic of the CLA toolkit is that it collects data from social media applications (e.g., Facebook and Twitter) to analyze student behaviour and student relationships. The authors applied the CLA toolkit in different "student-facing LA" case studies (Kitto, Lupton, Davis, \& Waters, 2017) to make learners reflect upon and change their learning behaviour.

Other informal learning infrastructures integrate tools and support LA without a pedagogical design. An example is the ROLE Sandbox ${ }^{3}$, a widget-based personal learning environment built on theories of self-regulated learning (Kravcik \& Klamma, 2012). In Renzel \& Klamma (2013), ROLE Sandbox log data is exploited to extract statistics and to define several social networks. The authors argue that directly analyzing web log data has the advantage of guaranteeing data interoperability among different services without needing to develop new data standards. This solution technically enables data sharing and processing, but web log data does not include any kind of learning concept, which hinders the integration and reuse of learning-specific information. In order to overcome this limitation, other technical frameworks and infrastructures have been proposed. Two interesting examples are the Contextualized Attention Metadata (CAM) framework (Schmitz, Wolpers, Kirschenmann, \& Niemann, 2011) and Learn-B (Siadaty et al., 2012). Their aim is to enhance the collection, processing, and offering of learning data. The CAM framework is used to log activities from those tools that generate CAM records; the logs can then be offered to analyze the learning process. The CAM framework has already been employed for several purposes, including learning object classification, competency detection, emotional state recognition, and goal and intention detection (see Schmitz et al. 2011). On the other hand, Learn-B is a service-based software environment designed to support self-regulated learning in the

\footnotetext{
${ }^{3}$ http: //role-sandbox.eu
} 
Table 1. Comparison of Several Infrastructures and Frameworks for LA

\begin{tabular}{|c|c|c|c|c|c|c|c|}
\hline & \multicolumn{2}{|c|}{ Functionality offered } & \multirow[b]{2}{*}{ Extensibility } & \multirow[b]{2}{*}{$\begin{array}{l}\text { Informal } \\
\text { learning }\end{array}$} & \multirow[b]{2}{*}{$\begin{array}{l}\text { Workplace } \\
\text { learning }\end{array}$} & \multicolumn{2}{|l|}{ Technical issues } \\
\hline & $\begin{array}{l}\text { Knowledge } \\
\text { metaphor }\end{array}$ & $\begin{array}{l}\text { Retrieves } \\
\text { data from }\end{array}$ & & & & Data model & API \\
\hline Fidalgo-Blanco et al. & K. creation & Moodle & Low & Partly & No & AAN & \\
\hline CLA toolkit & K. creation & Social Media & High & Partly & No & Ontology-based & JSON-LD xAPI \\
\hline ROLE Sandbox & Participation & ROLE widgets & Low & Yes & Partly & Social network & REST \\
\hline CAM framework & K. acquisition & Integrated tools & High & Yes & Partly & Ontology-based & \\
\hline Learn-B & K. acquisition & Integrated tools & High & Yes & Yes & Ontology-based & \\
\hline Apereo LAI & K. acquisition & xAPI tools & High & No & No & Ontology-based & xAPI \\
\hline Watershed LRS & K. acquisition & xAPI tools & Low & Yes & Partly & Ontology-based & xAPI \\
\hline SSS & K. creation & Integrated SSS & High & Yes & Yes & Context-aware AAN & REST \\
\hline
\end{tabular}

workplace. Learn-B was employed in several studies of the Intel-LEO project ${ }^{4}$ for several purposes, such as assessing the impact of scaffolding practices in workplace environments (Siadaty, Gašević, \& Hatala, 2016a,b). Following the knowledge acquisition metaphor of learning, both the CAM framework and Learn-B defined ontologies to structure activity logs. Both are also able to describe the contexts where the learning activities happen.

As several infrastructures were proposed, how to share data among them became a relevant problem. For this reason, open software and standards were promoted for LA (Siemens et al., 2011). In this regard, Experience API (xAPI) ${ }^{5}$ was adopted as a de facto standard for the exchange of learning data. Its main idea is to define a common data format between learning tools and infrastructures to exchange information about learning events. According to xAPI, each learning event is defined by a quadruple: a subject, a verb, an object, and a context, while an ontology should be implemented to define the different elements of the quadruple. As an example, Bakharia et al. (2016) use JSON for Linked Data (JSON-LD) to define an extensible vocabulary for XAPI statements.

Some learning record stores (LRSs) have also been proposed to collect and manipulate xAPI data. Examples of LRSs with an open licence are Learning Locker ${ }^{6}$ and Larissa ${ }^{7}$. Other initiatives have built on top of them to create open infrastructures or toolkits that can be adapted to different learning situations. Some examples are Starfish Analytics ${ }^{8}$, Jisc Learning Analytics ${ }^{9}$, and Apereo Learning Analytics Initiative (LAI) ${ }^{10}$ (we included Apereo LAI in Table 1 as an example to represent this group of LRSs). These proposals support the collection and storage of xAPI data. They also offer some data analysis services and some user interfaces (e.g., SNA algorithms and dashboards). These services and interfaces can be adapted to different learning situations, or new services can be integrated into the toolkit. However, all of these initiatives focus on formal learning and take an institutional perspective. An interesting example of an xAPI-compliant infrastructure that supports informal learning is Watershed LRS ${ }^{11}$. The data analysis of Watershed LRS again follows the knowledge acquisition approach in a rather individualistic way. However, it is a closed infrastructure, offered as a cloud service, that cannot be extended by third parties.

\section{The SSS}

The SSS (Dennerlein, Kowald, et al., 2015) is an infrastructure that collects data from workplace learning tools and offers it back to be used by LA applications. It evolved over eight years from a close analysis of workplace learning practices in different domains carried out in the MATURE project ${ }^{12}$ (Ravenscroft, Schmidt, Cook, \& Bradley, 2012). More recently, it has been applied in the Learning Layers project ${ }^{13}$ to support informal workplace learning (Ley et al., 2014) with a special focus on small and medium-size enterprises working in innovation-driven domains. Its theoretical roots lie in knowledge creation theories. Moreover, its design was based on a number of additional empirical studies, such as in-depth case studies of workplace and organizational learning (e.g., Kaschig et al., 2012) and a number of design-based research activities in several contexts (Dennerlein, Theiler, et al., 2015). These studies contributed to the understanding of how individual, group, and organizational learning are intertwined in knowledge creation. To name just a few examples, the studies found out how professionals make sense of experiences and informally learn from them (Dennerlein et al., 2014), how help-seeking happens in professional

\footnotetext{
${ }^{4}$ http://intelleo.eu/index.php

${ }^{5}$ https://experienceapi.com

${ }^{6}$ https://www.ht2labs.com/learning-locker-community/overview/

${ }^{7}$ https://github.com/Apereo-Learning-Analytics-Initiative/Larissa

${ }^{8}$ https://www.starfishsolutions.com/home/starfish-enterprise-success-platform/starfish-analytics/

${ }^{9}$ https://www.jisc.ac.uk/learning-analytics

10 https://www . apereo.org/communities/learning-analytics-initiative

${ }^{11}$ http: //www. watershedlrs.com

12 https://mature-ip.eu/

${ }^{13}$ http: //learning-layers.eu
} 


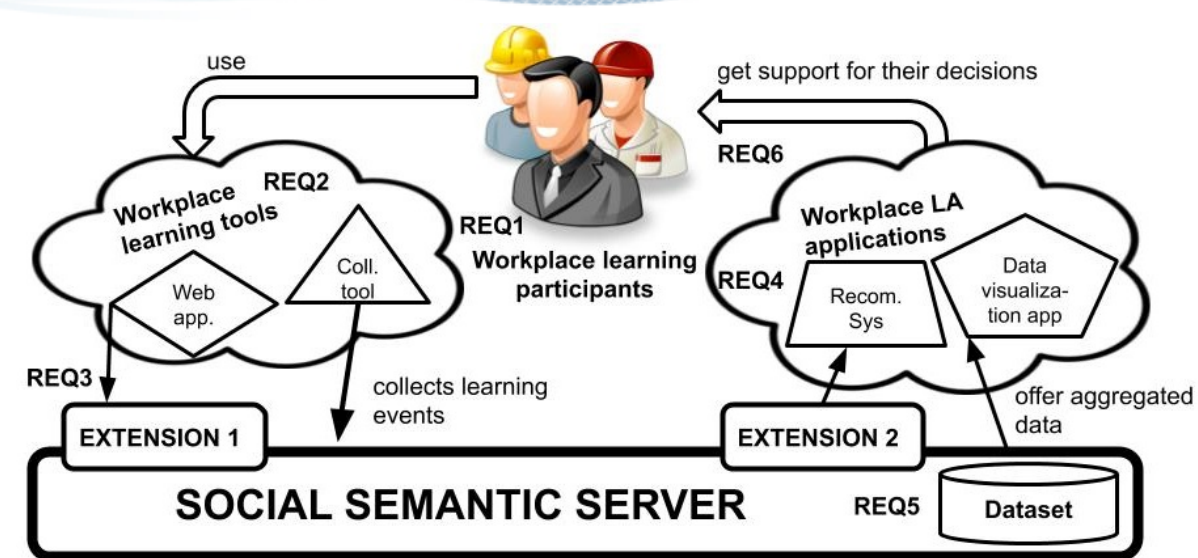

Figure 1. Potential scenario supported by the SSS

networks (Santos et al., 2016), and how organizations create boundary objects to facilitate knowledge sharing (Kaschig et al., 2012). The SSS was designed by deriving requirements from the tools and services needed to support these empirical studies.

\subsection{Requirements for the SSS}

Figure 1 depicts a typical scenario for a workplace LA infrastructure. Workplace learning participants (e.g., workers or trainers) use a set of tools to learn at the workplace. The workplace LA infrastructure collects the learning events from these tools and creates a coherent dataset out of them. This data is then offered back to workplace LA applications to support the decision making of workplace learning participants based on their learning evidence.

The SSS has evolved into an open-source infrastructure designed to address the knowledge creation metaphor for this type of scenario. It is not restricted to a specific domain or activity, so it should support a wide range of workplace learning scenarios (REQ3 in Table 2 and Figure 1), which may differ in the way they are enacted, in their number of participants, and in their level of formality (Kooken, Ley, \& De Hoog, 2007). Therefore, the SSS should be flexible enough to adapt to many different learning situations.

The SSS is meant to be used during normal activity in real work environments (REQ2). Hence, the SSS should be able to remove the inherent boundaries from the large variety of tools that are currently used for workplace learning (Kooken, Ley, $\&$ De Hoog, 2007). It is well known that in real work environments different tools are used for learning purposes (Cardinali, 2015). Hence, the SSS should collect the learning events tracked by different tools and integrate them (REQ1). It should also enable different integration strategies, because the technical aspects of these tools may also differ.

The data collected by the SSS should be offered back to LA applications later on (REQ4). Therefore, the SSS should offer a data-access API for external applications. It would also be desirable for the SSS to allow the definition of new data APIs. Thus, the wide range of LA applications that are currently used for workplace learning (Ruiz-Calleja, Prieto, Ley, Rodríguez-Triana, \& Dennerlein, 2017) could potentially exploit the SSS's data.

Other requirements related to how the SSS structures the knowledge derive from its focus on the knowledge creation metaphor (REQ5). As we showed in the previous section, the LA applications that follow this metaphor establish an AAN. This AAN should be able to describe different relationships between actors and artifacts (e.g., resource creation or access). It is key for this metaphor to track the context in which interactions between learners, and between learners and artifacts, take place. The special focus of knowledge creation on emerging knowledge also requires the SSS to represent different data structures in different levels of maturity (Ruiz-Calleja, Prieto, Ley, Rodríguez-Triana, \& Dennerlein, 2017). Finally, because the SSS is meant to support decision making in workplace learning practices (REQ6), exploitating the data collected by the SSS is expected to have a positive impact on these practices.

\subsection{Design and Implementation of the SSS}

\subsubsection{SSS Data Model}

The requirements of the SSS data model are derived from its focus on the knowledge creation metaphor (REQ5) and the need to support the semantic integration of data collected from different learning tools (REQ3) with different levels of formality (REQ1). The basis of the SSS data model is an AAN, in accordance with the analysis of LA applications that follows the knowledge creation metaphor. Therefore, the SSS can explicitly describe the relationships between learners, between artifacts, and between learners and artifacts. Furthermore, different types of relationships can be defined, giving meaning to the connections among entities. Some contextual information (e.g., time or some keywords) can also be attached to the entities. 


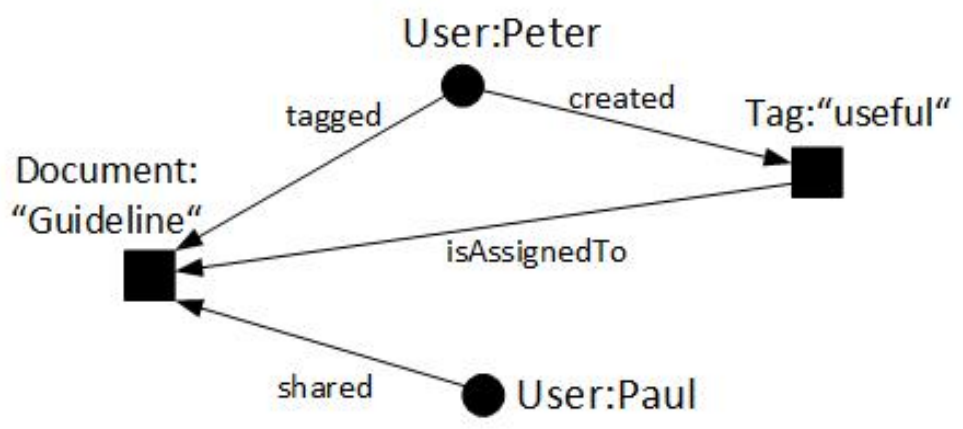

Figure 2. Example of an AAN from the SSS

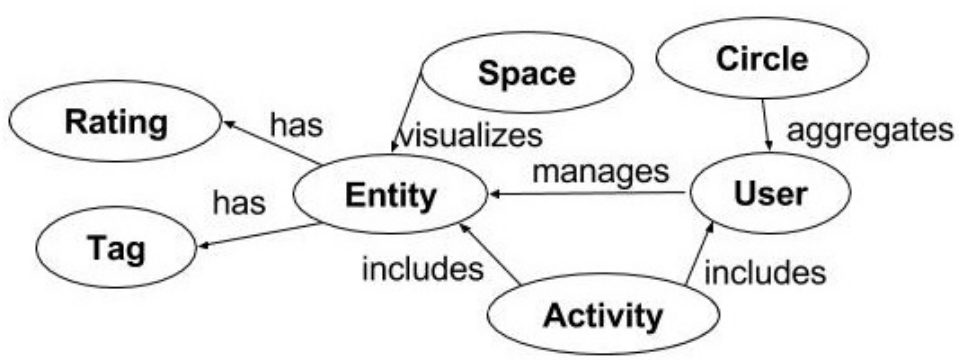

Figure 3. The SSS core ontology

As an example, a typical situation in informal learning is a worker (let's call him Paul) sharing a document that describes a guideline for a particular work process. A colleague (Peter) finds this document, marks it for his own use, and tags it as "useful." In this case, the AAN will register four entities (both users, the document, and the tag) and four relationships with three different meanings ("tagged," "shared," and "isAssignedTo"). In addition, the relationships between the entities will include the time frame of the event and maybe some contextual information, such as the location or the tool employed for the event. Figure 2 graphically depicts the resulting AAN from this example.

This AAN can be seen as a high-level abstraction that offers a common data model to integrate data from multiple learning tools. However, the entities related to the AAN (actors, artifacts, relationships, and contexts) should be semantically described if the semantic integration of data is required. For this reason, the SSS includes a core ontology. This ontology is used to describe the entities in the AAN, their relationships, and the parameters to define the context where these relationships happen. Hence, the data model of the SSS is based on a context-aware and semantically enriched AAN.

Figure 3 represents the entities and the most important relationships in the SSS core ontology. The main entities to define nodes in the AAN are User and Entity. Users can be aggregated into Circles (i.e., an abstraction similar to Google+ Circles, to aggregate users into groups) and entities into Spaces (i.e., an abstraction similar to Dropbox spaces, to aggregate documents into folders). Then, some metadata can be attached to the entities (Rating and Tag). Finally, the Activity is used to trace activities where users and entities are involved. Note that the SSS core ontology does not include a concept to define contexts because the parameters related to learning contexts highly depend on each specific situation. For example, in some situations, the context is related to the metadata attached to the entity (e.g., tags), while in others it is related to the spaces to which the entities are aggregated.

This ontology can be extended later on to include additional concepts for specific learning situations. For instance, in order to describe the AAN depicted in Figure 2, the concept Document should be defined as a subclass of Entity, and the relationship Shared should also be defined. In some other cases, ontology extensions are used to define parameters to describe learning contexts, such as location. It should be noted that the SSS faces a well-known trade-off: ontologies are more expressive vocabularies than folksonomies and are used to model domains or to allow the semantic integration of several applications; however, ontologies are sometimes not able to collect emerging knowledge because they are more difficult to modify and evolve. For this reason, ontology extensions can provide further structure by semantically defining narrower concepts (e.g., different types of "artifacts"), but this semantic definition can also be avoided (e.g., by defining a folksonomy of tags). Thus, the SSS can integrate data with different levels of formality (Ruiz-Calleja, Dennerlein, Tomberg, Ley, et al., 2015). 


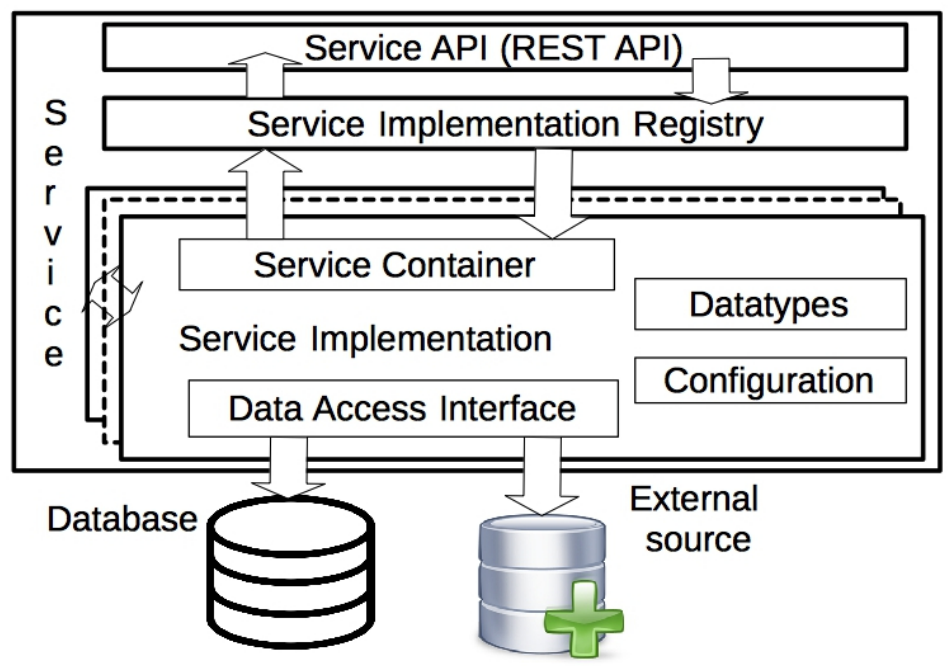

Figure 4. SSS service architecture

\subsubsection{SSS Software Architecture and Implementation}

The SSS software infrastructure should allow the integration of a wide variety of tools used for learning in the workplace (REQ3 and REQ2). It should also be flexible, to be adapted to different informal learning situations (REQ1), and extensible, so new functionalities can be offered to data-consuming applications (REQ4).

The SSS software architecture follows the service-oriented-architecture (SOA) style (Earl, 2005). SOA promotes architectures based on the light integration of loosely coupled services that offer a granular functionality and can be orchestrated to provide a more complex functionality. SOA leads to flexible and modular architectures, since services can be exchanged if needed. This is achieved by dividing the functionality of the SSS into fine granular services that can be easily maintained, reused, combined, and replaced. Thus, by adding new services or configuring the existing ones, the SSS can be extended to offer additional functionalities or it can be adapted to specific learning scenarios.

Figure 4 depicts the architecture of each SSS service. Each service comprises a set of Service Implementations, a Service Implementation Registry, and a Service API. Each service may include several Service Implementations (or just one) that offer the same functionalities in different ways. For example, a tag recommendation service may have two implementations, each of them based on different recommendation algorithms. Each service may define its own Datatypes and can have some Configuration parameters. In the previous example, the configuration parameters define the data sources accessed by the implementations of the recommender service or the way each result is ordered. This way, it is possible to modify the internal logic of the services (and adapt their functionality accordingly) without needing to change the Service API. Each Service Implementation also includes a Data Access Interface, which is used to access data sources. These data sources can be databases integrated into the SSS (e.g., MySQL) or external data sources (e.g., external applications that share their data with the SSS). The Service Implementation Registry mediates the communication between the Service API and the Service Implementations, deriving each API call to the corresponding implementation through its Service Container.

Figure 5 represents a possible configuration of the SSS software architecture. It can be seen that external learning tools submit their data to the SSS by calling the Activity service, which traces the interaction between learners and resources. The data is then stored by the Metadata services (e.g., Data export service), which manage the datasets of the SSS. These Metadata services also offer abstractions - based on the AAN previously described — for other services to access the data. Thus, the data APIs implemented in the Metadata services wrap the interfaces of the underlying databases, which may vary depending on the data representation used (e.g., SQL, NoSQL, or document-based representations). Thus, the implementation of the data model can be abstracted to fit the business logic of each service. This solution makes the SSS data layer scalable and adaptable. However, this solution does not require good data access performance. This is because learning datasets are typically smaller than domains, and current data stores offer enough data access performance. While it is true that the implementation of the Metadata services may hinder the performance because it adds an extra software layer, our previous experience (see the first paragraph of section 3) shows that this does not represent a problem for users or software developers. Finally, the business logic of the SSS is composed of another collection of services. They can be Simple services, which serve one functionality (e.g., Group Access Restriction, which controls user restrictions) or manage some entity types (e.g., Tag, Collections, Q\&A, or Activity). Others are Composed services, which 


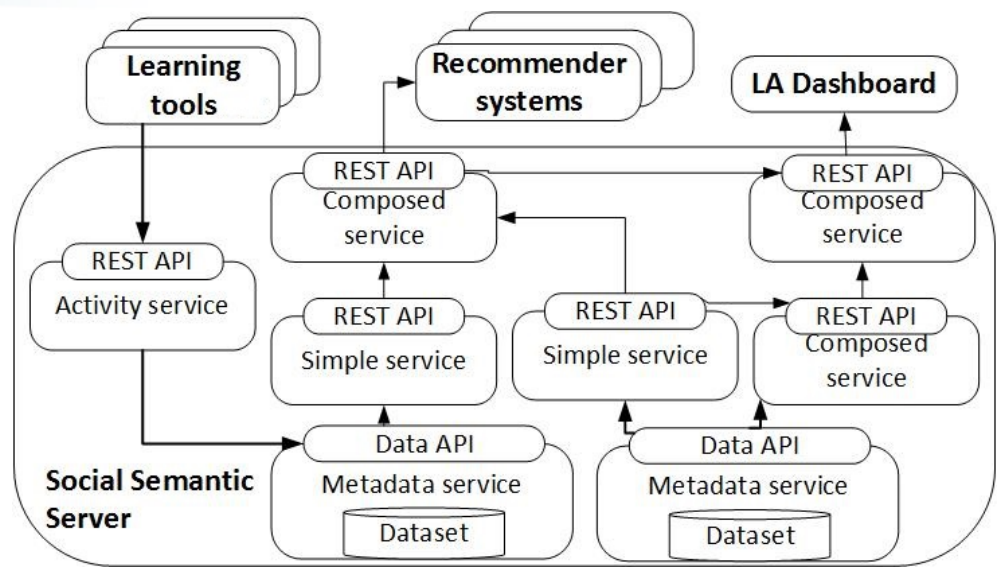

Figure 5. Possible configuration of the SSS software architecture

exploit other services to provide their own functionality (e.g., Search or Recommendations). See Dennerlein, Kowald, et al. (2015) for additional details about these services.

As previously seen, the design of the SSS architecture promotes its flexibility and extensibility. Services can be composed, and different implementations or configurations of the services can coexist. This way, the SSS can be adapted to different workplace learning situations. Furthermore, the SSS includes a set of services that can be exploited by several LA functionalities, such as the Activity service or the Metadata services, while the Service API is offered as a REST API.

Regarding the integration of learning tools and LA applications, the SSS supports two strategies. The first one is a loosely coupled integration, where external applications make use of the SSS data API either to publish or to retrieve data. In these cases, the functionality of the SSS does not need to be extended or modified, although an extension of the SSS may be required to enable the semantic integration of the data retrieved (i.e., new concepts may need to be defined). The second strategy is a tightly coupled integration, where part of the functionality of the applications is developed as SSS services or as extensions of already-existing ones. It is sometimes the case that these new services need to define new API methods to offer new functionalities that could potentially be reused by other applications. Therefore, the SSS can be seen as a framework that facilitates the development of workplace LA applications (Dennerlein, Kowald, et al., 2015).

The implementation of the SSS architecture is based on microservices (Newman, 2005), which have emerged as a novel way to design software in the form of services to promote independence in their own deployment and to deal with technological heterogeneity. This independence of the services increases the flexibility of the SSS infrastructure because some of them can be deployed and others can be integrated if needed. Furthermore, the loose coupling of SSS services also enables their development by third parties and the integration of software frameworks inside the SSS. In fact, the current version of the SSS (which is coded in Java and available in our GitHub repository ${ }^{14}$ ) integrates the TagRec (Kowald, Kopeinik, \& Lex, 2017) framework for the development of tag recommender algorithms.

\section{Evaluation}

In order to assess whether the SSS meets the requirements stated in the previous section, we developed a set of workplace learning tools that submit their data to the SSS, as well as LA applications that consume this data. We then used the SSS in authentic evaluation studies carried out in the Learning Layers project. Note that carrying out authentic studies that involve different stakeholders (Dewan, 2001) is typically done to evaluate collaborative systems in education (e.g., Alario-Hoyos et al. 2013). Table 2 summarizes the SSS requirements and their relationships with the evaluation studies, the evaluation methods, and the data sources.

\subsection{Workplace Learning Tools Integrated into the SSS}

We integrated into the SSS some domain-independent tools offered by third parties during the Learning Layers project. These tools were extended to submit data to the SSS or to make use of some of its services. One example is the web browser Chrome ${ }^{15}$. We developed a Chrome plugin (called Bookmarker (Ruiz-Calleja, Dennerlein, Tomberg, Ley, et al., 2015)) that allows us to create, tag, and submit bookmarks to the SSS from a Chrome interface. We followed a similar strategy to integrate the blog editor WordPress ${ }^{16}$ with an extension called Attacher (Ruiz-Calleja, Dennerlein, Tomberg, Ley, et al., 2015). Attacher allows

\footnotetext{
${ }^{14}$ https://github.com/learning-layers/Socialsemanticserver

15 https: / / www.google.com/chrome/

16 https: //wordpress.com
} 
Table 2. Relationship between SSS Requirements, Evaluation Studies, Evaluation Methods and Data Sources

\begin{tabular}{lllll}
\hline Tag & Requirement & Evaluation studies & Evaluation methods & Data sources \\
\hline \hline \multirow{2}{*}{ REQ1 } & $\begin{array}{l}\text { Supports LA in a wide range of } \\
\text { learning scenarios }\end{array}$ & $\begin{array}{l}\text { TT-14, CC-15, } \\
\text { MR-15, and RW-16 }\end{array}$ & $\begin{array}{l}\text { Multiple authentic studies, } \\
\text { mixed methods }\end{array}$ & $\begin{array}{l}\text { SSS logs, interviews, } \\
\text { questionnaires }\end{array}$ \\
\hline \multirow{2}{*}{ REQ2 } & $\begin{array}{l}\text { Integrates tools used } \\
\text { for learning in the workplace }\end{array}$ & $\begin{array}{l}\text { TT-14, CC-15 } \\
\text { MR-15, and RW-16 }\end{array}$ & $\begin{array}{l}\text { Multiple authentic studies, } \\
\text { feature analysis }\end{array}$ & $\begin{array}{l}\text { Implementation of the SSS, } \\
\text { SSS logs, LA applications }\end{array}$ \\
\hline \multirow{2}{*}{ REQ3 } & $\begin{array}{l}\text { Collects and integrates data } \\
\text { from different tools }\end{array}$ & $\begin{array}{l}\text { TT-14, MR-15, } \\
\text { and RW-16 }\end{array}$ & Multiple authentic studies & SSS logs \\
\hline \multirow{2}{*}{ REQ4 } & $\begin{array}{l}\text { Enables the consumption } \\
\text { of its data by LA applications }\end{array}$ & $\begin{array}{l}\text { LA applications } \\
\text { development }\end{array}$ & Feature analysis & LA applications \\
\hline \multirow{2}{*}{ REQ6 } & $\begin{array}{l}\text { Focuses on knowledge } \\
\text { creation metaphor }\end{array}$ & $\begin{array}{l}\text { TT-14 } \\
\text { and CC-15 } \\
\text { zing and supporting learning practices }\end{array}$ & $\begin{array}{l}\text { Multiple authentic studies, } \\
\text { mixed methods, feature analysis }\end{array}$ & $\begin{array}{l}\text { Implementation of the SSS, } \\
\text { interviews, LA applications }\end{array}$ \\
\hline
\end{tabular}

blog editors to browse the resources (e.g., bookmarks) contained in the SSS from the blog editing interface, to access their corresponding URLs, and to cite them in the blog posts. Attacher also registers in the SSS the blog posts published. A different strategy was followed to integrate Evernote ${ }^{17}$ (Dennerlein, Kowald, et al., 2015). In this case, an SSS service was created to access Evernote API in order to semantically integrate into the SSS the notes taken by its users, turning Evernote into an external data source from which data could be imported to the SSS.

We also developed from scratch other learning tools that make use of the SSS. In these cases, the SSS was exploited as a framework for the development of workplace learning tools that are tightly integrated into it (Dennerlein, Kowald, et al., 2015). This approach was followed by KnowBrain (Dennerlein, Theiler, et al., 2015), a collaborative resource-hosting tool built on top of the SSS. KnowBrain exploits the SSS services to allow users to manage, tag, and share resources, such as learning documents or bookmarks. Similarly, a set of three tools was developed to support informal learning in the health care domain, which is typically combined in one tool set that uses the SSS as a common back-end infrastructure. The first tool is Bits \& Pieces (Dennerlein et al., 2014), a visual categorization tool to enhance individual and collaborative sense-making processes; it exploits the SSS to enable learners to define semantic and contextualized relationships between learning artifacts. The second tool is Discussion Tool, a question and answer tool that offers an interface similar to a web forum (Dennerlein et al., 2014); it exploits the SSS to relate the resources created (questions and replies) to each other and these resources to their authors and readers. The third tool is Living Documents (Bachl, Zaki, Schmidt, \& Kunzmann, 2014), a collaborative text editor; it exploits the SSS to allow its users to access the resources registered in the infrastructure.

\subsection{LA Applications That Exploit the SSS}

In addition to the learning tools mentioned in the previous subsection, we developed a set of LA applications that exploit the data collected by the SSS. Specifically, we developed a visual dashboard and five recommender services, which offer typical functionalities of workplace LA (Ruiz-Calleja, Prieto, Ley, Rodríguez-Triana, \& Dennerlein, 2017). The development of a bigger set of applications that offer other workplace LA functionalities is part of our future work.

\subsubsection{SSS Dashboard}

The SSS Dashboard (Ruiz-Calleja, Dennerlein, Ley, \& Lex, 2016) allows end users to visualize and browse the data collected by the SSS. Specifically, the SSS Dashboard contains three visualizations: filter events, which represents the list of events collected by the AAN and allows users to filter them by their actors, by the artifacts involved, or by the actions done; Social network, which represents a social network of the artifact-mediated relationships between the actors registered by the SSS; and Tag Cloud, which represents a tag cloud of the tags registered in the AAN.

These visual abstractions are suitable for learners and trainers to visualize "uptake relations," which have been defined as the smallest units of meaning-making activities in knowledge creation (Suthers \& Dwyer, 2014). For example, Figure 6 shows how the Dashboard represents these meaning-making activities by building a social network that is generated from the uptake relations arising from reciprocal contributions to a particular artifact. These uptake activities represent the use and reuse of certain tags when collecting and annotating learning materials. The directional social network, therefore, represents the subgroups of learners that have collaborated around the creation, use, and enrichment of concepts. It also represents the subgroup of learners who have influenced others in this process.

The SSS Dashboard was implemented as a loosely coupled application that consumes the data offered by the SSS. It simply gathers a .csv file from the Data Export Service API that contains the events registered in the AAN. This

\footnotetext{
${ }^{17}$ https: //evernote.com/
} 
Table 3. Main Characteristics of the SSS Evaluation studies

\begin{tabular}{|c|c|c|c|c|c|c|c|c|}
\hline Tag & Date & Location & Duration & Domain & Purpose & Users & Tools & LA applications \\
\hline TT-14 & 2014 & Estonia & 5 months & Education & $\begin{array}{l}\text { Reflect on how to introduce } \\
\text { technology in education }\end{array}$ & 11 & $\begin{array}{l}\text { Chrome, } \\
\text { WordPress }\end{array}$ & SSS Dashboard \\
\hline CC-15 & 2015 & Austria & 4 weeks & Research & Study state of the art & 18 & KnowBrain & 3Layers, MostPopular \\
\hline MR-15 & 2015 & England & 2 months & $\begin{array}{l}\text { Medical } \\
\text { practice }\end{array}$ & $\begin{array}{l}\text { Make sense of their } \\
\text { working experience }\end{array}$ & 6 & $\begin{array}{l}\text { Evernote, Bits \& Pieces, } \\
\text { Discussion Tool, } \\
\text { Living Documents }\end{array}$ & $\begin{array}{l}\text { MostPopular, CF } \\
\text { recommendation } \\
\text { algorithm }\end{array}$ \\
\hline RW-16 & 2016 & Europe & 5 months & Research & $\begin{array}{l}\text { Plan and evaluate } \\
\text { interventions }\end{array}$ & 22 & $\begin{array}{l}\text { Evernote, Bits \& Pieces, } \\
\text { Discussion Tool, } \\
\text { Living Documents }\end{array}$ & $\begin{array}{l}\text { MostPopular, CF } \\
\text { recommendation } \\
\text { algorithm }\end{array}$ \\
\hline
\end{tabular}

implementation decision is not as efficient as extending the Data Export Service with a CRUD REST API to offer abstraction suitable for the Dashboard. However, we reduced our development time because we reused the Data Export Service extension developed for the recommendation algorithms (see next subsection). The current version of the Dashboard was implemented using Javascript and well-known libraries, such as $\mathrm{d} 33^{18}$.

\subsubsection{Recommendation Algorithms}

Other LA applications that exploit the data collected by the SSS are recommender services that take advantage of the TagRec (Kowald, Kopeinik, \& Lex, 2017) framework integrated into the SSS. As part of this evaluation, we developed a set of recommendation algorithms that exploit the SSS data for different purposes: to recommend learning resources (Seitlinger et al., 2015), to recommend tags to be assigned to learning resources, and to recommend people to interact with (Kopeinik, Kowald, Hasani-Mavriqi, \& Lex, 2017).

Several recommendation algorithms are currently available. These include tag and resource recommendations based on recent or most popular resources (Kowald \& Lex, 2016; Kowald, Seitlinger, Trattner, \& Ley, 2014). Thus, these approaches rank the resources in the learning system based on popularity or recency (i.e., time since last usage). Another recommendation approach is based on collaborative filtering (CF) (Schafer, Frankowski, Herlocker, \& Sen, 2007), which means that items of similar users are recommended. In the future, we also plan to extend this CF-based approach with a content-based approach as provided in the ScaR recommender framework (Lacic, Traub, Kowald, \& Lex, 2015), which would allow us to incorporate similarities not only between users but also between resources (e.g., based on description texts or even full contents).

An algorithm that is especially designed to follow knowledge creation theory is the 3Layers tag recommendation approach (Seitlinger, Kowald, Trattner, \& Ley, 2013; Kowald, Seitlinger, Kopeinik, Ley, \& Trattner, 2013). It learns how a learner, or group of learners, categorizes resources. It traces the tags used for particular resources; later on, it dynamically builds an understanding of the semantic contexts over time. The algorithm then recommends tags that match a particular semantic context. This mirrors a situation where the interpretation of a resource (its semantic context) emerges from past artifact-mediated activity, rather than being predefined by an ontology of the domain.

The recommender services were implemented ${ }^{19}$ as a microservice in the SSS (Recommendations) service, which includes the TagRec framework. The Data Export Service was also extended to dynamically create a .csv file with the structure required by the framework, out of the data contained in the SSS database. This extension of the Data Export Service to offer the data as a .csv file did not take efficiency as a requirement. However, it managed to wrap the data contained in the SSS database to be offered to a service that takes a .csv file as an input without needing to modify the service. The Recommendations microservice also defines new methods in the SSS API. As an example, it includes a method that provides a set of recommended tags whose parameters are a user, a set of entities, a category, and a maximum number of tags to recommend. External applications (e.g., KnowBrain and Bits \& Pieces) exploit this method to provide tag recommendations to their users.

\subsection{Evaluation Studies}

Due to the complexities of introducing and evaluating workplace learning technology, it was not feasible to evaluate all requirements of the infrastructure in a single study. Hence, we conducted four evaluation studies to collect evidence for the six requirements described in the previous section. These studies cover different domains and different goals and happened in different countries. We focused on uptake events and on how the infrastructure traced and supported them, as these are clear indications of collaborative knowledge building. Table 3 summarizes the most important characteristics of the evaluation studies, while further information is provided next.

\footnotetext{
${ }^{18}$ http://d3js.org/

${ }^{19}$ https://github.com/learning-layers/TagRec/
} 


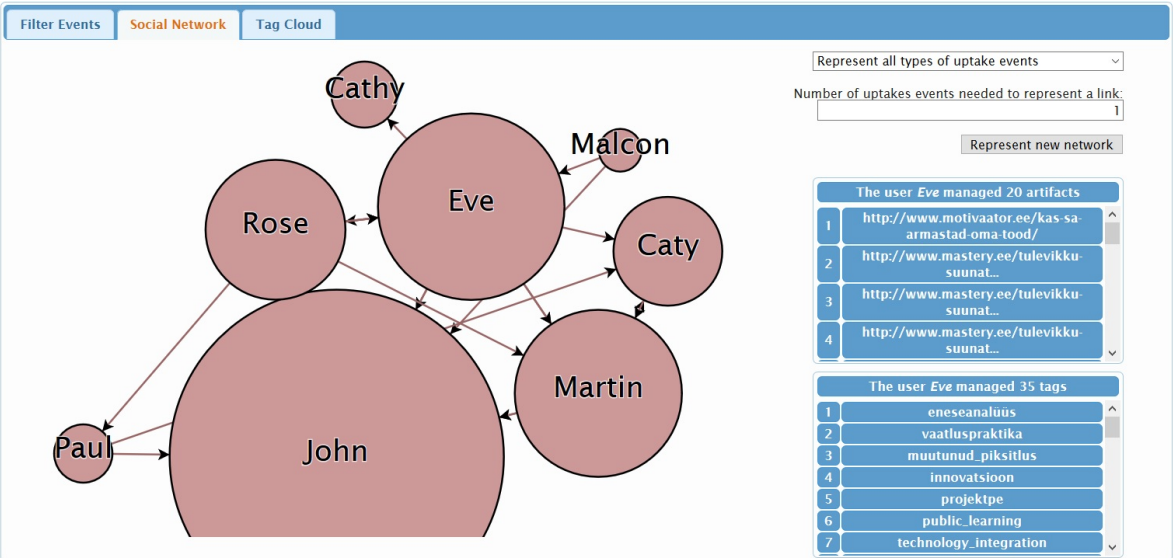

Figure 6. SSS Dashboard interface: social network visualization from the data collected in TT-14

\subsubsection{Professional Teacher Training Course (TT-14)}

The first evaluation study was done in a professional teacher training course (TT-14 in Tables 2 and 3) held at Tallinn University (Estonia) between September 2014 and January 2015. The study tested the SSS Dashboard in an authentic experience where learners used multiple workplace learning tools (REQ3 and REQ2) and evaluated the Dashboard's support for understanding the learning process (REQ1 and REQ6) according to the knowledge creation metaphor (REQ5). Here we focus on the role played by the SSS, while additional details are reported in Ruiz-Calleja et al. (2016).

Learning context and participants: The main purpose of the course is to help teachers reflect on how to introduce new technologies and pedagogical techniques into their classrooms. Therefore, they had to address real problems and opportunities in their workplace. A group of 10 professional teachers ("learners" from now on) attended the course and were guided by a trainer. As part of the course activities, the learners were asked to browse the web looking for resources that could extend or contrast the information provided by the trainer. Using these resources, the learners were asked to write blog posts where they reflected about their own teacher practice and how to introduce new technology in their own classrooms. Each learner wrote 10 blog posts, which they shared with the rest of the learners. They used Chrome as a web browser to search and discover web resources that would help them design their teaching. These resources were submitted as bookmarks to the SSS with the support of the Bookmarker extension. They used WordPress as a blog editor. The Attacher plugin was installed to facilitate access to bookmarks published on the SSS.

SSS configuration: A simple configuration of the SSS was required for this study because all the learning tools and LA applications are loosely integrated. The service Tag was exploited by Attacher and Bookmarker to submit learning resources, attaching to them some contextual metadata. Attacher also uses the Search service to search for resources, and the SSS Dashboard uses the Data Export Service to extract the AAN from the SSS. Regarding the data model, a folksonomy of tags was created during the study, but there was no need to define new semantic concepts. Hence, it was not necessary to extend the core ontology of the SSS.

Data collection and analysis: Once the training course was over, one of the learners and the trainer used the SSS Dashboard to visualize the data collected by the SSS. Figure 6 represents the social network interface of the SSS Dashboard (the names of the learners were changed). The functionality of the SSS Dashboard was explained to the trainer and the learner, and, after that, six tasks were proposed for them to accomplish with the Dashboard. The tasks emerged from the learning procedures defined by the three learning metaphors (e.g., "Detect which interests two learners have in common" or "Identify learning topics that are surprising or unexpected to you"). Once they finished, a semi-structured interview was carried out to further understand their opinion about the dashboard and how useful they found the graphical abstractions offered (e.g., "If the SSS Dashboard was available during the course, what would you use it for?"). The interactions of the trainer and the learner with the SSS Dashboard, their voice while using it, and the interviews were recorded. Later on, two researchers listened to the recordings and extracted their most important aspects.

Resulting AAN: During five months, eight of the learners frequently used Chrome and WordPress, while the other two hardly used these tools since they were not active in the course. The SSS coherently combined the data from Chrome and WordPress, and a total of 320 events were registered. Out of these events, the SSS created an AAN that contained 11 actors, 53 resources, and 116 tags.

Main study results: Both the trainer and the learner were able to accomplish the six tasks proposed using the SSS Dashboard. Both of them agreed that by using it they could better understand the learning process. Interestingly, they understood the collaboration among learners as a process mediated by artifacts (trainer while using the Dashboard: "as a 
trainer I would be worried because these two learners did not reuse artifacts from others and they do not share information with others"). During the interview, both of them agreed that the SSS Dashboard was a useful application. However, the trainer found it interesting to understand the learning process (trainer when interviewed: "an average trainer would use it to understand what is going on in the course"), while the learner reduced its potential use to identifying relevant learning artifacts or finding potential collaborators (learner when interviewed: "I would use the dashboard to find out if there are learners that use the same resources as me and to get an overview of the resources used by others").

\subsubsection{Collaborative Digital Curation (CC-15)}

The second evaluation study was conducted as part of a collaborative digital curation scenario (CC-15) coordinated from Austria in September 2015. The aim of this study was to test the tag recommender services in a real work environment (REQ1 and REQ2) and to compare the support provided by the frequency-based MostPopular algorithm and the 3Layers algorithm (REQ5), as well as to evaluate the benefit offered to the participants (REQ6). Part of the results were published in Seitlinger et al. (2017).

Learning context and participants: As part of their job, 18 professional researchers ("users" from now on) explored the topic "smart workplaces" over four weeks in order to collaboratively write a state-of-the-art overview. These researchers belonged to two universities from two European countries. They coordinated among themselves to collect and share topicrelevant resources (i.e., web pages or text documents), sharing at least four every week. For this purpose they used KnowBrain, which allowed them to upload, classify, tag, and share resources. Each resource was related to a category (out of six predefined ones) that classified the resources according to their topics, and each was annotated with free tags. The users were supported in the process of tagging resources by a tag recommender service integrated into KnowBrain, which suggested seven tags to describe each resource depending on the category selected for the resource. These recommendations were extracted from two algorithms that exploited the data from the SSS: either 3Layers or the frequency-based MostPopular. One of the assumptions of the study was that 3Layers recommendations would be more suitable for creative group work because they would pick up and feed back emergent topics more quickly than MostPopular recommendations.

SSS configuration: KnowBrain is a learning application tightly integrated into the SSS whose complex functionality uses the following SSS services (Dennerlein, Kowald, et al., 2015): Recommendations, Gardening Knowledge Structures, Collections, Q\&A, Search, Tag, Data Export Service, Activity, and Group Access Restriction. KnowBrain submits data to the SSS through the Collections, Q\&A, and Tag services. This data is later on exploited by the recommender services (recommendations service). The recommender services extract the AAN from the data export service and offer the tag recommendations through an extension of the SSS API. Additionally, the SSS core ontology was extended to define two new types of entities ( $\mathrm{f} i \mathrm{l}$ e and image). Two other concepts were introduced: collection, to aggregate entities, and friend, to allow explicit relationships between users.

Data collection and analysis: We analyzed the KnowBrain and SSS logs to understand the tag recommendations and selections during the study. We measured the support of each recommendation algorithm by means of the F1 score (Power, 2011), which is calculated based on the number of accepted recommendations of a tag and the number of times the tag was recommended to the users. We used the F1 score because it is a simple and commonly employed metric that considers both the precision and the recall of the recommendations, providing a more robust evaluation procedure. We also analyzed the timestamps of the log entries to understand how the tag assignments evolved in KnowBrain and which tags were uptaken by which users.

Resulting AAN: Eighteen users participated in the study. A total of 2,654 user events were registered, out of which the SSS created an AAN that contained 18 actors, 122 resources, 263 unique tags, 701 relationships between tags and resources, and 6 categories. The recommender services made extensive use of this AAN; there were 183 recommendation events with a total of 1,281 tags recommended.

Main study results: The score obtained by the 3Layers algorithm (F1-score $=0.34$ ) was higher than the score obtained by the frequency-based MostPopular algorithm $(\mathrm{F} 1$-score $=0.27)$. These results show that the recommendations offered by the 3Layers algorithm have a bigger influence on the users' behaviour than the popularity-based algorithm. We assume that this was the case because 3Layers better reflected the shared interpretations that emerged in the group as a result of their creative group work. We could also notice its impact by the capability of the 3Layers algorithm to raise awareness of topics once they are introduced into the users' community. For example, the tags wellbeing and social involvement were marginal at the time they were introduced. These tags represented new concepts introduced in the learning community and were recommended by the 3Layers algorithm (and not by the MostPopular algorithm), causing them to be quickly taken up by other users.

\subsubsection{Meaning Making for Health Care Professionals (MR-15)}

The third evaluation study was part of a three-year design-based research project that aimed at developing workplace learning tools for interdisciplinary health care professionals. It was conducted at the participants' daily work from October to November 2015. The aim of the study was to assess whether the SSS can collect and integrate data from multiple tools (REQ3) that are 
employed to support informal learning while working (REQ1 and REQ2). The benefit of the data collected to the participants will also be assessed (REQ6).

Learning context and participants: The study involved six English health care professionals (two doctors, one practice manager, one office supervisor, one administrator, and one IT support manager). During the study, the health care professionals used a tool set that included four tools integrated into the SSS. These tools supported the collection, categorization, and formalization of informal learning experiences: Evernote helped record informal learning experiences by taking multimedia notes; Bits \& Pieces facilitated making sense of the notes taken with Evernote; Discussion Tool supported parallel discussions and promoted professional engagement in sense-making processes; and Living Documents supported the formalization of the conclusions. In addition, Bits \& Pieces exploited two of the recommender algorithms integrated into the SSS: a timebased recommender, which recommended tags to annotate learning artifacts, and a collaborative filtering algorithm, which recommended resources (e.g., Evernote notes) created by other professionals.

SSS configuration: In this study, the SSS configuration is more complex because four learning tools are involved. For the tight integration of Bits \& Pieces, two new services (Learning Episode and Category) were needed. These services categorize and contextualize learning resources in an abstraction called Learning Episode, defined as a new entity type in the SSS ontology. Discussion Tool is also tightly integrated into the SSS. It exploits services such as $Q \& A, E n t i t y$, and Tag. Although no new service was needed for this integration, the SSS ontology was extended with the entity type Discussion and the relationship Like. The other two applications involved simply registered in the SSS the documents managed by their users. For this purpose, two new services were required: Data Import to access Evernote API and Living Document to retrieve documents written by Living Document. These services also defined new entity types: Mail, Evernote note, and LivingDoc.

Data collection and analysis: We conducted questionnaires and semi-structured interviews with the professionals to evaluate the impact of the recommender services on supporting informal learning. The questionnaire addressed the appropriateness of functionalities and asked for their evaluation in the form of open questions; the interviews focused on how the tools were used and on their impact on learning and working. We analyzed the data following Mayring's inductive qualitative content analysis (Mayring, 2014): first, we transcribed the interviews; second, we paraphrased the contents; and third, we extracted categories to iteratively discover structure such as use cases, working functionalities, and corresponding reasoning in the data. We also analyzed the SSS logs to describe the resulting AAN and to quantify the tag recommendations.

Resulting AAN: The SSS registered 8,345 user events and 151 resources imported from Evernote and Bits \& Pieces. The resulting AAN contained 6 actors, 306 resources (145 bits used in episodes, 29 discussions related to 36 discussion entries from Discussion Tool, 13 documents from Living Documents, and 48 learning episodes from Bits \& Pieces), 31 tags, and 71 categories. Based on this AAN, tag recommendations were computed, which led to 12 accepted recommendations.

Main study results: In the analysis of the qualitative data, we found out that the users all followed the informal learning process from tracing experiences, organizing and discussing them, and finally transforming outcomes into a shared report. The support of the SSS as an infrastructure to share, categorize, and evolve annotated learning resources among four different tools was key for the learning process. In addition, the SSS integrated the data collected from the four tools and offered it to the recommender algorithms. However, these recommender algorithms did not play a major role in this particular scenario. One of the reasons is that the resource recommendations were happening on a personal level by having bits added by colleagues rather than using the ones provided by the system. The infrequent use of the tagging functions and the limited number of tags registered in the system lowered the impact of the tag recommendations. We claim that the infrequent use is due to users' lack of experience with similar tools and functionalities, which hides the additional value of tagging resources. However, some of the users appreciated the tags when searching for resources (health care professional: "I use tags and things like that because I think obviously if [...] everybody's got a lot more diverse tags it would be very useful to search on; I like that"). Despite this, the professionals stated in the interviews that the recommendation of tags eases the process of tagging and that they can see how the tags can help them arrive at a professionally agreed upon vocabulary.

\subsubsection{Collaborative Planning of Research Work (RW-16)}

The fourth evaluation study was also part of the three-year design-based research project reported in MR-15. In this case, the SSS supported a group of researchers from March to July 2016 to coordinate their work related to the planning of research interventions, evaluation of the data gathered, and reflection on the results obtained. In a similar way as the MR-15 pilot, we assessed whether the SSS could collect and integrate data from multiple tools (REQ3) used to support informal learning while working (REQ1 and REQ2). We also evaluated the impact of the data collected to support users' learning practice (REQ6).

Learning context and participants: This study involved 22 researchers belonging to 10 different institutions and collaborating on a European project. During the study, the researchers used the SSS and the same tool set described in the MR-15 pilot study. They used Evernote, Bits \& Pieces, Discussion Tool, and Living Documents to communicate and to coordinate their activities. These tools supported the individual and collaborative collection, sense-making, sharing, and formalization of ideas.

SSS configuration: The SSS configuration is exactly the same as in the MR-15 evaluation study. 
Data collection and analysis: As was the case in the MR-15 pilot, we quantitatively analyzed the SSS logs to describe the resulting AAN and to quantify the tag recommendations. We also analyzed the log files to detect uptake events where tags were introduced and reused by another user. Thus, we assessed how the recommender services supported collaborative learning.

Resulting AAN: The SSS registered 182,911 user events and 293 resources imported from Evernote and Bits \& Pieces. The resulting AAN contained 40 actors, 1,056 resources (259 bits used in episodes, 75 discussions related to 96 discussion entries from Discussion Tool, 49 documents from Living Documents, and 29 learning episodes from Bits \& Pieces), 688 tag assignments of a total of 301 distinct tags, and 132 categories. Based on this, AAN tag recommendations were computed, which led to 102 accepted recommendations.

Main study results: The log analysis shows that the researchers followed a similar learning process as in the MR-15 pilot study: tracing experiences, making sense of and discussing them, and transforming outcomes into reports. Again, the SSS infrastructure provided a key support to this learning process as it allowed learners to share data and documents across the applications. In this pilot study, the learners made extensive use of tags. These tagging events were facilitated by the recommender service, which also promoted the uptake of tags. In fact, 88 tag-uptake events were registered involving 67 unique tags. As we have shown in previous studies, these uptake events can facilitate the establishment of shared understanding in the sense-making process (Dennerlein, Seitlinger, Lex, \& Ley, 2016; Ley \& Seitlinger, 2015).

\subsection{Discussion of the Evaluation Findings}

\subsubsection{Accomplishment of the SSS Requirements}

Supports informal and formal scenarios from different domains (REQ1): The diversity of learning and LA applications integrated into the SSS allows it to support LA in a wide range of workplace learning scenarios and contexts. In fact, our evaluation studies range from formal scenarios in a professional training course (TT-14) to completely informal others (MR-15 and RW-16) where participants had the opportunity to use the learning tools in authentic working conditions. The flexibility of the SSS - promoted by its microservice-based architecture and its data model — was key to supporting these scenarios, because different services and ontology extensions were required.

Integrates tools used for learning in the workplace (REQ2): The SSS collects and integrates data from workplace learning tools that differ both in their functional and in their technical characteristics. These tools include general-purpose and independent ones that exploit the Activity Service (e.g., WordPress (TT-14)), those that require a new service for collecting their data (e.g., Evernote (MR-15 and RW-16)), and purpose-specific tools. The different integration strategies again promoted by the microservice-based architecture of the SSS — and the flexibility of the SSS data model made it possible to collect data from a wide variety of tools.

Collects and integrates data (REQ3): The evaluation studies TT-14, MR-15, and RW-16 included learning processes where the SSS collected and coherently integrated data from multiple learning tools to offer it back to LA applications. The data model managed by the SSS played a major role in the data integration: the AAN is a flexible structure that allows it to relate different concepts, while the SSS core ontology offered a high-level abstraction common to many learning tools that enabled it to describe the entities of the AAN.

Supports LA applications (REQ4): The development of LA applications showed how the data collected by the SSS can be exploited by LA applications. We exemplified this by integrating a visual dashboard and a set of recommender algorithms. These LA applications not only offer different functionalities but also follow different approaches to consuming the data from the SSS: while the SSS Dashboard was implemented as an external application that simply retrieves the SSS data from the Data Export Service API, the recommender algorithms were implemented as microservices that extend the SSS functionality and offer a new data API that other applications (KnowBrain and Bits \& Pieces) exploit. Again, the SSS flexibility was key to enabling several integration strategies for its data consumption. It is also noteworthy that, due to the reduced number of participants, these studies do not assess whether the LA applications could exploit larger amounts of data collected by the SSS. However, a previous study (Kowald et al., 2015) shows how the 3Layers and MostPopular algorithms could satisfactorily exploit data from 10,000 users imported to the SSS from several social tagging systems.

Supports the knowledge creation metaphor (REQ5): The studies we conducted offer clear evidence for the focus on the knowledge creation metaphor by the SSS to support workplace LA. In TT-14, the SSS Dashboard supported learners and trainers to understand the learning process from the knowledge creation point of view. Both types of users identified how artifacts were co-developed and how knowledge emerged inside the community. We obtained the first evidence that trainers appreciated the visualization of shared artifacts and how learners contributed to these artifacts.

CC-15 showed that the 3Layers recommender algorithm provided more accurate results than the MostPopular algorithm, which offered the most popular results in a certain period. 3Layers also facilitated the introduction of new concepts into the community of learners because it also recommended newly introduced tags even if they were not the most common tags. 3Layers recommendations therefore better reflected the situation of creative group work (Seitlinger et al., 2017), which is evidence of the algorithm's foundation in knowledge creation theories. 
In MR-15 and RW-16, the infrastructure supported knowledge maturation as it helped learners move through a collaborative learning process across several tools. Learners started with the collection of ideas, then could share them, and finally were able to formalize them. The SSS, and more specifically its data model, played a central role in making objects of activity available across tools and in allowing the tracing of interactions with these objects: first, the explicit relationships between the entities enabled the detection of uptake events and co-creation processes; second, the flexibility of the AAN allowed it to introduce new artifacts and tags and to dynamically learn the participants' shared categories over time. The limited number of participants in the evaluation studies limits the generalizability of these findings to larger communities of learners; it would be interesting to assess the support provided by the SSS to knowledge building in organizations, or even in cross-organizational networks, where innovation is more likely to happen.

Provides data that is relevant for analyzing and supporting learning practices (REQ6): Finally, we exemplified the impact of the SSS on workplace learning practices. In TT-14, the data facilitated the comprehension of the learning process by a trainer and a learner. Since they only used the SSS Dashboard once the course was over, it did not have a direct influence on this process. However, they understood the Dashboard as a useful application, and the trainer explicitly said that she would have intervened if she had seen it during the course. In CC-15, the SSS data influenced the users when tagging resources, helping them to co-create conceptual artifacts and to introduce new ones into the learning community. The tag recommender 3Layers, based on knowledge creation, achieved higher acceptance than the one based on popularity only, MostPopular. In MR-15, the SSS supported a two-year design-based research project that resulted in new tools and practices for health care professionals. The SSS allowed individuals to collect and formalize learning experiences, share them, and work collaboratively on them. Due to the limited use of the tagging system and the resource recommendations, the SSS data had little impact on the users in this study. On the other hand, RW-16 showed how an informal learning process supported by a similar technical configuration was enhanced by the tag recommender services. However, it remains to be seen whether recommender services are an effective way to exploit the data. Another open question is how to increase their impact on workplace learning processes, especially on the more informal ones that entail a larger number of learners. In this regard, we are currently starting a large-scale study on the use of the recommender services in the health care domain.

\subsubsection{An AAN to Support the Knowledge Creation Metaphor}

We started this paper from the assumption that a technical infrastructure that supports LA needs to start from an understanding of what learning is, how it takes place, and how it can be supported. The SSS has its roots in the conception of learning as a knowledge-building and maturing process, and it is therefore rooted in an understanding of knowledge as a dynamic and social process of co-construction and creation (Ravenscroft, Schmidt, Cook, \& Bradley, 2012). The evaluation studies that we have conducted show several knowledge creation and maturing activities that have been traced and supported. Our experience also shows that the knowledge creation metaphor provides a special challenge for the design of LA infrastructures. Such infrastructures should offer enough flexibility—both in their software architecture and in their data model— to allow for knowledge to emerge from individual and collaborative activities.

Uptake has been described as the smallest unit in collaborative learning that can be observed as an interaction (Suthers \& Dwyer, 2014). In our evaluation studies, uptake occurred at several places. In RW-16, for example, several learners contributed ideas to shared collections. Reuse of tags occurred in CC-15 and RW-16. A powerful analytic framework has recently been created that allows interactions to be analyzed in "contingency graphs" (Suthers \& Dwyer, 2014). With the uptake framework, a big step has been taken toward making collaborative learning activity traceable for LA. While the uptake framework exists as an analytical tool that allows post hoc analysis of learning data, with the SSS, we provide the technical means to trace and represent uptake events in life systems and feed back this data to learners and trainers.

Once the infrastructure became focused on challenging scenarios of knowledge creation, we found it easier to support use cases that are more in line with other metaphors. For example, the SSS also includes recommender services that are more related to knowledge acquisition, such as time-based recommenders, or participation, such as collaborative filtering. Another example is the exploitation of SSS data to create a social network graph representation that shows community membership and centrality of members (Ruiz-Calleja, Dennerlein, Tomberg, Pata, et al., 2015), following the participation metaphor.

\section{Conclusions and Future Work}

This paper proposes to exploit the SSS as an infrastructure for workplace LA that follows the knowledge creation metaphor. For this purpose, we first derived the requirements for an infrastructure that complies with knowledge creation theories. We then designed and developed the SSS as a microservice-based infrastructure whose data model is based on a semantically enriched and context-aware AAN. Its evaluation entailed the integration of several learning tools that submit their data to the SSS and some LA applications - a visual dashboard and a set of recommender services - that exploit its data. This evaluation also comprised four authentic workplace learning scenarios where 57 participants were satisfactorily supported by the SSS.

Two characteristics of the SSS are key for its support of workplace LA. First, the flexibility of its software architecture allows 
it to adapt to a wide range of learning scenarios by configuring the microservices that are deployed. It also allows different integration strategies for learning tools and LA applications. Thus, it is possible to collect data from external tools and to develop new services to provide additional functionality (e.g., tag recommendation) that can be exploited by external LA applications. The second key characteristic is the SSS's data model. The AAN allows the model to describe contextualized uptake events. Furthermore, the AAN allows the model to describe the entities of the AAN with different degrees of formality, allowing both the semantic integration of data, using ontologies, and the description of emerging knowledge, using folksonomies.

Our research suffers from the limitations that are common when addressing learning at a workplace. Because workers learn in a self-directed way, any technology that supports this learning needs to seamlessly fit existing practices that may vary dramatically in different domains and organizations. Hence, samples sizes of studies are usually small, and it is often difficult to assess how technology might be adopted in large networks of learners. We addressed these challenges by employing an iterative, design-based research strategy that collects evidence over a number of iterations and across different contexts. In this paper, we reported on four such studies. Together with the previous empirical studies that we have also mentioned, these should allow us to build up converging evidence of the utility of our general approach over time, although the scalability of our proposal reminds to be assessed.

Our future work will focus on further exploiting the SSS for workplace LA in real scenarios. We are currently working on a large-scale study that includes the use of recommenders in the health care domain. We will also promote the development of a wider collection of LA applications that make use of the SSS data. We plan to encourage the community of learning tool developers to adopt the SSS by making it compliant with xAPI. xAPI could be supported by developing a new Metadata service and a new Activity service. The development of such services, and their corresponding APIs, would be facilitated if we used as a dataset an LRS with an open licence. Finally, we will explore the potential use of the SSS data to support additional LA services, such as the automatic detection of learning needs in communities of workers.

\section{Declaration of Conflicting Interest}

The authors declared no potential conflicts of interest with respect to the research, authorship, and/or publication of this article.

\section{Funding}

This research has been partially funded by the FP7 ICT Workprogramme of the European Community: "Learning Layers Scaling up Technologies for Informal Learning in SME Clusters" (grant no. 318209) and by the European Union's Horizon 2020 research and innovation program: "CEITER — Cross-Border Educational Innovation through Technology-Enhanced Research" (grant no. 669074) and "AFEL — Analytics for Everyday Learning” (grant no. 687916). It has also been partially funded by the Know-Center GmbH Graz (Austrian FFG COMET Program).

\section{References}

Alario-Hoyos, C., Bote-Lorenzo, M., Gómez-Sánchez, E., Asensio-Pérez, J., Vega-Gorgojo, G., \& Ruiz-Calleja, A. (2013). GLUE! An architecture for the integration of external tools in virtual learning environments. Computers \& Education, 60(1), 122-137. https://dx.doi.org/10.1016/j.compedu.2012.08.010

Bachl, M., Zaki, D., Schmidt, A. P., \& Kunzmann, C. (2014). Living documents as a collaboration and knowledge maturing platform. In Proceedings of the 14th International Conference on Knowledge Technologies and Data-Driven Business ( $i$-KNOW 14), 16-19 September 2014, Graz, Austria (pp. 30:1-30:4). New York: ACM. https://dx.doi.org/10.1145/2637748.2638437

Bakharia, A., Kitto, K., Pardo, A., Gašević, D., \& Dawson, S. (2016). Recipe for success: Lessons learnt from using xAPI within the Connected Learning Analytics toolkit. In Proceedings of the 6th International Conference on Learning Analytics and Knowledge (LAK '16), 25-29 April 2016, Edinburgh, UK (pp. 378-382). New York: ACM. https://dx.doi.org/10.1145/2883851.2883882

Berendt, B., Vuorikari, R., Littlejohn, A., \& Margaryan, A. (2014). Learning analytics and their application in technologyenhanced professional learning. In A. Littlejohn \& A. Margaryan (Eds.), Technology-Enhanced Professional Learning: Processes, Practices and Tools (pp. 144-157). Abingdon-on-Thames, UK: Routledge.

Buckingham-Shum, S., \& Ferguson, R. (2012). Social learning analytics. Journal of Educational Technology \& Society, 15(3), 3-26.

Cardinali, F. (2015). Towards Learning Analytics Interoperability at the Workplace (LAW Profile). Learning Analytics Review no. 5 (Tech. Rep. No. ISSN: 2057-7494). Retrieved from http://www.laceproject.eu/learning-analytics-review/law-interoperability/

de Laat, M., \& Schreurs, B. (2013). Visualizing informal professional development networks. Building a case for learning analytics in the workplace. American Behavioral Scientist, 57(10), 1421-1438. https://dx.doi.org/10.1177\%2F0002764213479364 
Dennerlein, S., Kowald, D., Lex, E., Theiler, D., Lacic, E., Ley, T., ... Ruiz-Calleja, A. (2015). The Social Semantic Server: A flexible framework to support informal learning at the workplace. In Proceedings of the 15th International Conference on Knowledge Technologies and Data-Driven Business (I-KNOW 2015), 21-23 October 2015, Graz, Austria (pp. 26:1-26:8). New York: ACM. https://dx.doi.org/10.1145/2809563.2809614

Dennerlein, S., Rella, M., Tomberg, V., Theiler, D., Treasure-Jones, T., Kerr, M., .. Trattner, C. (2014). Making sense of Bits and Pieces: A sensemaking tool for informal workplace learning. In Proceedings of the 9th European Conference on Technology Enhanced Learning (EC-TEL 2014), 16-19 September 2014, Graz, Austria (pp. 391-397). Lecture Notes in Computer Science, Springer. https://dx.doi.org/10.1007/978-3-319-11200-831

Dennerlein, S., Seitlinger, P., Lex, E., \& Ley, T. (2016). Take up my tags: Exploring benefits of meaning making in a collaborative learning task at the workplace. In Proceedings of the European Conference on Technology Enhanced Learning (EC-TEL 2016), 13-16 September 2016, Lyon, France (pp. 377-383). Lecture Notes in Computer Science, Springer.

Dennerlein, S., Theiler, D., Marton, P., Santos Rodriguez, P., Cook, J., Lindstaedt, S., \& Lex, E. (2015). KnowBrain: An online social knowledge repository for informal workplace learning. In Proceedings of the European Conference on Technology Enhanced Learning (EC-TEL 2015), 15-17 September 2015, Toledo, Spain (pp. 509-512). Lecture Notes in Computer Science, Springer. https://dx.doi.org/10.1007/978-3-319-24258-348

Derntl, M., Günnemann, N., \& Klamma, R. (2013). A dynamic topic model of learning analytics research. In Proceedings of the LAK Data Challenge, Held at the 3rd International Conference on Learning Analytics and Knowledge (LAK'13), 8-12 April 2013, Leuven, Belgium (pp. 1-5). New York: ACM.

Dewan, P. (2001). An integrated approach to designing and evaluating collaborative applications and infrastructures. Computer Supported Cooperative Work, 1(10), 75-111. https://dx.doi.org/10.1023/A:1011266229161

Duval, E. (2011). Attention please! Learning analytics for visualization and recommendation. In Proceedings of the 1st International Conference on Learning Analytics and Knowledge (LAK'11), 27 February-1 March 2011, Banff, AB, Canada (pp. 9-17). New York: ACM.

Earl, T. (2005). Service-Oriented Architecture: Concepts, Technology and Design. Upper Saddle River, NJ, USA: Prentice Hall PTR.

Eraut, M. (2004). Informal learning in the workplace. Studies in Continuing Education, 26(2), 247-273. https://dx.doi.org/10.1080/158037042000225245

Fidalgo-Blanco, A., Sein-Echaluce, M. L., Garcia-Penalvo, F. J., \& Conde, M. A. (2015). Using learning analytics to improve teamwork assessment. Computers in Human Behavior, 47(1), 149-156. https://dx.doi.org/10.1016/j.chb.2014.11.050

Gašević, D., Dawson, S., \& Siemens, G. (2015). Let's not forget: Learning analytics are about learning. TechTrends, 59(1), 64-71. https://dx.doi.org/10.1007/s11528-014-0822-x

Kaschig, A., Maier, R., Sandow, A., Brown, A., Ley, T., Magenheim, J., ... Seitlinger, P. (2012). Technological and organizational arrangements sparking effects on individual, community and organizational learning. In Proceedings of the 7th European Conference on Technology-Enhanced Learning (EC-TEL 2012), 18-21 September 2012, Saarbrücken, Germany (pp. 180-193). Lecture Notes in Computer Science, Springer.

Kitto, K., Lupton, M., Davis, K., \& Waters, Z. (2017). Designing for student-facing learning analytics. Australasian Journal of Educational Technology, 33(5), 152-168. https://dx.doi.org/https://doi.org/10.14742/ajet.3607

Klamma, R. (2013). Community learning analytics - Challenges and opportunities. In Proceedings of the 12th International Conference on Web-Based Learning (ICWL 2013), 6-9 October 2013, Kenting, Taiwan (pp. 284-293). Springer. https://dx.doi.org/10.1007/978-3-642-41175-529

Kooken, J., Ley, T., \& De Hoog, R. (2007). How do people learn at the workplace? Investigating four workplace learning assumptions. In Proceedings of the 2nd European Conference on Technology Enhanced Learning (EC-TEL 2007), 17-20 September 2007, Crete, Greece (pp. 158-171). Heidelberg, Germany: Springer. https://dx.doi.org/10.1007/978-3-540-75195-3,2

Kopeinik, S., Kowald, D., Hasani-Mavriqi, I., \& Lex, E. (2017). Improving collaborative filtering using a cognitive model of human category learning. The Journal of Web Science, 2(4), 45-61. https://dx.doi.org/10.1561/106.00000007

Kowald, D., Kopeinik, S., \& Lex, E. (2017). The TagRec framework as a toolkit for the development of tag-based recommender systems. In Adjunct Publication of the 25th Conference on User Modeling, Adaptation and Personalization, 9-12 July 2017, Bratislava, Slovakia (pp. 23-28). New York: ACM. https://dx.doi.org/10.1145/3099023.3099069

Kowald, D., Kopeinik, S., Seitlinger, P., Ley, T., Albert, D., \& Trattner, C. (2015). Refining frequency-based tag reuse predictions by means of time and semantic context. In Mining, Modeling, and Recommending “Things” in Social Media (pp. 55-74). Springer.

Kowald, D., \& Lex, E. (2016). The influence of frequency, recency and semantic context on the reuse of tags in social tagging systems. In Proceedings of the 27th ACM Conference on Hypertext and Social Media, 10-13 July 2016, Halifax, NS, Canada (pp. 237-242). New York: ACM. https://dx.doi.org/10.1145/2914586.2914617 
Kowald, D., Seitlinger, P., Kopeinik, S., Ley, T., \& Trattner, C. (2013). Forgetting the words but remembering the meaning: Modeling forgetting in a verbal and semantic tag recommender. In Mining, Modeling, and Recommending "Things" in Social Media (pp. 75-95). Springer.

Kowald, D., Seitlinger, P., Trattner, C., \& Ley, T. (2014). Long time no see: The probability of reusing tags as a function of frequency and recency. In Proceedings of the 23rd International Conference on World Wide Web (WWWC 2014), 7-11 April 2014, Seoul, Korea (pp. 463-468). New York: ACM. https://dx.doi.org/10.1145/2567948.2576934

Kravcik, M., \& Klamma, R. (2012). Supporting self-regulation by personal learning environments. In Proceedings of the 12th International Conference on Advanced Learning Technologies (ICALT 2012), 7-10 July 2012, Rome, Italy (pp. 710-711). IEEE. https://dx.doi.org/10.1109/ICALT.2012.192

Krull, E., \& Leijen, A. (2015). Perspectives for defining student teacher perfomance-based teaching skills indicators to provide formative feedback through learning analytics. Creative Education, 6(10), 914-926. Retrieved from $10.4236 /$ ce. 2015.610093

Lacic, E., Traub, M., Kowald, D., \& Lex, E. (2015). ScaR: Towards a real-time recommender framework following the microservices architecture. In Proceedings of the Workshop on Large Scale Recommender Systems (LSRS2015) at RecSys 2015, 16-20 September 2015, Vienna, Austria (Vol. 15). Retrieved from https://pure.tugraz.at/ws/portalfiles/portal/3521071/RecSys ${ }_{L} \operatorname{SRS}_{2} 015 . \mathrm{pdf}$

Ley, T., Cook, J., Dennerlein, S., Kravcik, M., Kunzmann, C., Pata, K., ... Trattner, C. (2014). Scaling informal learning at the workplace: A model and four designs from a large-scale design-based research effort. British Journal of Educational Technology, 45(6), 1036-1048. https://dx.doi.org/10.1111/bjet.12197

Ley, T., \& Kump, B. (2013). Which user interactions predict levels of expertise in work-integrated learning? In Proceedings of the 8th European Conference on Technology-Enhanced Learning (EC-TEL 2013), 17-21 September 2013, Paphos, Cyprus (pp. 178-190). Lecture Notes in Computer Science, Springer. https://dx.doi.org/10.1007/978-3-642-40814-415

Ley, T., \& Seitlinger, P. (2015). Dynamics of human categorization in a collaborative tagging system: How social processes of semantic stabilization shape individual sensemaking. Computers in Human Behavior, 51, 140-151. https://dx.doi.org/10.1016/j.chb.2015.04.053

Mayring, P. (2014). Qualitative Content Analysis: Theoretical Foundation, Basic Procedures and Software Solution (Tech. Rep.). Klagenfurt, Austria: SSOAR.

Newman, S. (2005). Building Microservices. Designing Fine-Grained Systems. Sebastopol, CA, USA: O'Reilly Media.

Niemann, K., \& Wolpers, M. (2014). Usage-based clustering of learning resources to improve recommendations. In Proceedings of the 9th European Conference on Technology-Enhanced Learning (EC-TEL 2014), 16-19 September 2014, Graz, Austria (pp. 317-330). Lecture Notes in Computer Science, Springer. Retrieved from 10.1007/978-3-319-11200-824

Nonaka, I. (1994). A dynamic theory of organizational knowledge creation. Organization Science, 5(1), 14-37.

Nussbaumer, A., Berthold, M., Dahrendorf, D., Schmitz, H., Kravcik, M., \& Albert, D. (2012). A mashup recommender for creating personal learning environments. In Proceedings of the 11th International Conference on Web-Based Learning (ICWL 2012), 2-4 September 2012, Sinaia, Romania (pp. 79-88). Lecture Notes in Computer Science, Springer. Retrieved from 10.1007/978-3-642-33642-39

Paavola, S., \& Hakkarainen, K. (2005). The knowledge creation metaphor - An emergent epistemological approach to learning. Science \& Education, 14(6), 535-557. Retrieved from 10.1007/s11191-004-5157-0

Peschl, M. F., \& Fundneider, T. (2014). Designing and enabling spaces for collaborative knowledge creation and innovation: From managing to enabling innovation as socio-epistemological technology. Computers in Human Behavior, 37, 346-359. https://dx.doi.org/10.1016/j.chb.2012.05.027

Power, D. (2011). Evaluation: From precision, recall and F-measure to ROC, informedness, markedness and correlation. Journal of Machine Learning Technologies, 2(1), 37-63.

Rajagopal, K., van Bruggen, J. M., \& Sloep, P. B. (2017). Recommending peers for learning: Matching on dissimilarity in interpretations to provoke breakdown. British Journal of Educational Technology, 48(2), 385-406. https://dx.doi.org/10.1111/bjet.12366

Ravenscroft, A., Schmidt, A., Cook, J., \& Bradley, C. (2012). Designing social media for informal learning and knowledge maturing in the digital workplace. Journal of Computer Assisted Learning, 28(3), 235-349. https://dx.doi.org/10.1111/j.1365-2729.2012.00485.x

Renzel, D., \& Klamma, R. (2013). From micro to macro: Analyzing activity in the ROLE sandbox. In Proceedings of the 3rd International Conference on Learning Analytics and Knowledge (LAK'13), 8-12 April 2013, Leuven, Belgium (pp. 250-254). New York: ACM. https://dx.doi.org/10.1145/2460296.2460347

Ruiz-Calleja, A., Dennerlein, S., Ley, T., \& Lex, E. (2016). Visualizing workplace learning data with the SSS Dashboard. In CrossLAK 2016: International Workshop on Learning Analytics across Physical and Digital Spaces, 25 April 2016 , Edinburgh, UK (pp. 79-86). Retrieved from http://ceur-ws.org/Vol-1601/CrossLAK16Paper15.pdf 
Ruiz-Calleja, A., Dennerlein, S., Tomberg, V., Ley, T., Theiler, D., \& Lex, E. (2015). Integrating data across workplace learning applications with a social semantic infrastructure. In Proceedings of the 14th International Conference on Web-Based Learning (ICWL 2015), 5-8 November 2015, Guangzhou, China (pp. 208-217). Lecture Notes in Computer Science, Springer. https://dx.doi.org/10.1007/978-3-319-25515-6, 9

Ruiz-Calleja, A., Dennerlein, S., Tomberg, V., Pata, K., Ley, T., Theiler, D., \& Lex, E. (2015). Supporting learning analytics for informal workplace learning with a social semantic infrastructure. In Proceedings of the 10th European Conference on Tecnology Enhanced Learning (EC-TEL '15), 15-17 September 2015, Toledo, Spain (pp. 634-637). Lecture Notes in Computer Science, Springer. https://dx.doi.org/10.1007/978-3-319-24258-376

Ruiz-Calleja, A., Prieto, L., Ley, T., Rodríguez-Triana, M., \& Dennerlein, S. (2017). Learning analytics for professional and workplace learning: A literature review. In Proceedings of the European Conference on Technology Enhanced Learning (EC-TEL 2017), 12-15 September 2017, Tallinn, Estonia (pp. 164-178). Lecture Notes in Computer Science, Springer. https://dx.doi.org/10.1007/978-3-319-66610-5,3

Santos, P., Dennerlein, S., Theiler, D., Cook, J., Treasure-Jones, T., Holley, D., ... Lex, E. (2016). Going beyond your personal learning network, using recommendations and trust through a multimedia question-answering service for decision-support: A case study in the healthcare. Journal of Universal Computer Science, 22(3), 340-359. https://dx.doi.org/10.3217/jucs-022-03-0340

Schafer, J. B., Frankowski, D., Herlocker, J., \& Sen, S. (2007). Collaborative filtering recommender systems. In The Adaptive Web: Methods and Strategies of Web Personalization (pp. 291-324). Lecture Notes in Computer Science, Springer Berlin Heidelberg. https://dx.doi.org/10.1007/978-3-540-72079-99

Schmidt, A., Hinkelmann, K., Ley, T., Lindstaedt, S., Maier, R., \& Riss, U. (2009). Conceptual foundations for a service-oriented knowledge and learning architecture: Supporting content, process and ontology maturing. In S. Schaffert, K. Tochtermann, \& T. Pellegrini (Eds.), Networked Knowledge - Networked Media: Integrating Knowledge Management, New Media Technologies and Semantic Systems (pp. 79-94). Springer. https://dx.doi.org/10.1007/978-3-642-02184-86

Schmitz, H. C., Wolpers, M., Kirschenmann, U., \& Niemann, K. (2011). Contextualized attention metadata. In C. Roda (Ed.), Human Attention in Digital Environments (pp. 186-209). New York: ACM. https://dx.doi.org/10.1017/CBO9780511974519.008

Schoefegger, K., Seitlinger, P., \& Ley, T. (2010). Towards a user model for personalized recommendations in work-integrated learning: A report on an experimental study with a collaborative tagging system. In Proceedings of the 1st Workshop on Recommender Systems for Technology Enhanced Learning (RecSysTEL 2010), 30 September 2010, Barcelona, Spain (Vol. 1, pp. 2829-2838). Amsterdam, Holland: Elsevier. https://dx.doi.org/10.1016/j.procs.2010.08.008

Seitlinger, P., Kowald, D., Kopeinik, S., Hasani-Mavriqi, I., Lex, E., \& Ley, T. (2015). Attention please! A hybrid resource recommender mimicking attention-interpretation dynamics. In Proceedings of the 24th International Conference on World Wide Web, 18-22 May 2015, Florence, Italy (pp. 339-345). New York. https://dx.doi.org/10.1145/2740908.2743057

Seitlinger, P., Kowald, D., Trattner, C., \& Ley, T. (2013). Recommending tags with a model of human categorization. In Proceedings of the 22nd ACM International Conference on Information \& Knowledge Management (CIKM 2013), 27 October-1 November 2013, San Francisco, CA, USA (pp. 2381-2386). New York: ACM. https://dx.doi.org/10.1145/2505515.2505625

Seitlinger, P., Ley, T., Kowald, D., Theiler, D., Hasani-Mavriqi, I., Dennerlein, S., .. Albert, D. (2017). Balancing the fluency-consistency trade-off in collaborative information search with a recommender approach. International Journal of Human Computer Interaction, 34(6), 557-575. https://dx.doi.org/10.1080/10447318.2017.1379240

Siadaty, M., Gašević, D., \& Hatala, M. (2016a). Associations between technological scaffolding and micro-level processes of self-regulated learning: A workplace study. Computers in Human Behavior, 55B(1), 1007-1019. https://dx.doi.org/10.1016/j.chb.2015.10.035

Siadaty, M., Gašević, D., \& Hatala, M. (2016b). Measuring the impact of technological scaffolding interventions on micro-level processes of self-regulated workplace learning. Computers in Human Behavior, 59(1), 469-482. https://dx.doi.org/10.1016/j.chb.2016.02.025

Siadaty, M., Gašević, D., Jovanović, J., Milikić, N., Jeremić, Z., Ali, L., .. Hatala, M. (2012). Learn-B: A social analytics-enabled tool for self-regulated workplace learning. In Proceedings of the 2nd International Conference on Learning Analytics and Knowledge (LAK'12), 29 April-2 May 2012, Vancouver, Canada (pp. 115-119). New York: ACM. https://dx.doi.org/10.1145/2330601.2330632

Siemens, G., Gašević, D., Haythornthwaite, C., Dawson, S., Buckingham Shum, S., Ferguson, R., ... Baker, R. (2011). Open Learning Analytics: An Integrated and Modularized Platform (Tech. Rep.). Society for Learning Analytics Research (SOLAR). (https://solaresearch.org/wp-content/uploads/2011/12/OpenLearningAnalytics.pdf)

Southavilay, V., Yacef, K., Reimann, P., \& Calvo, R. A. (2013). Analysis of collaborative writing processes using revision maps and probabilistic topic models. In Proceedings of the 3rd International Conference on Learning Analytics and Knowledge (LAK '13), 8-12 April 2013, Leuven, Belgium (pp. 38-47). New York: ACM. (10.1145/2460296.2460307) 
Suthers, D., \& Dwyer, N. (2014). Multilevel analysis of uptake, sessions, and key actors in a socio-technical network. In Proceedings of the Computational Approaches to Connecting Levels of Analysis in Networked Learning Communities Workshop at Learning Analytics and Knowledge, 24-28 March 2014, Indianapolis, IN, USA (pp. 1-6). New York: ACM. https://dx.doi.org/http://ceur-ws.org/Vol-1137/LAK14CLA ubmission $_{1} . p d f$

Thüs, H., Chatti, M. A., Brandt, R., \& Schroeder, U. (2015). Evolution of interests in the learning context data model. In Proceedings of the 10th European Conference on Technology Enchanced Learning (EC-TEL 2015), 15-17 September 2015, Toledo, Spain (pp. 479-484). Lecture Notes in Computer Science, Springer. https://dx.doi.org/10.1007/978-3-319-24258-343 